\title{
Effects of Autonomic Blockade on Self-Similarity of Blood Pressure and Heart Rate Time Series
}

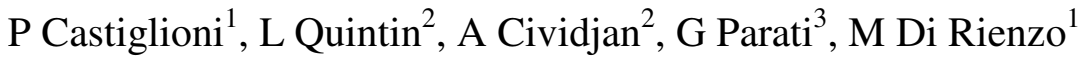 \\ ${ }^{1}$ Centro di Bioingegneria, Fondazione Don C. Gnocchi ONLUS, Milan, Italy \\ ${ }^{2}$ Physiologie (CNRS UMR 5123), School of Biology, University Lyon 1, Lyon, France \\ ${ }^{3}$ University of Milano-Bicocca, Italy and Istituto Auxologico Italiano, Milan, Italy
}

\begin{abstract}
Little is known on the effects of autonomic alterations on the characteristics of self-similarity of cardiovascular time series. To clarify this issue, changes in scaling exponents for blood pressure and heart rate following sympathetic and parasympathetic blockades were studied. We recorded $R R$ interval (RRI) and mean arterial pressure (MAP) beat by beat in 8 volunteers before and after administration of: propranolol, a non specific $\beta 1$ and $\beta 2$ cardiac and vascular antagonist; clonidine, an $\alpha 2$ agonist; and atropine, blocker of cardiac vagal activity. Self-similarity coefficients for RRI, $H_{R R I}$, and MAP, $H_{M A P}$, were estimated by detrended fluctuations analysis. Under baseline condition, $H_{M A P}$ was significantly greater than $H_{R R}$. Sympathetic blockades reduced $H_{R R I}$ only, while vagal blockade increased both $H_{R R I}$ and $H_{M A P}$. Results revealed differences in the scaling structures of MAP and $R R I$, and in the way their self-similarity is influenced by the autonomic regulation.
\end{abstract}

\section{Introduction}

In the last two decades, spectral analysis of blood pressure and heart rate has allowed to investigate several physiological and pathological aspects of the autonomic control of circulation. More recently, a number of studies focussed on complex and nonlinear characteristics of the cardiovascular dynamics. In particular, fractal-like anatomical structures in the heart and vasculature, and complex interactions among vascular beds, suggested that the cardiovascular signals are generated by fractals, selfsimilar processes, which lack a characteristic scale of time $[1,2]$. Therefore the study of blood pressure and heart rate self-similarity is considered a promising tool for deepen our knowledge on the cardiovascular regulation.

However, although it has been already reported that the self-similarity characteristics of heart rate may change with age or specific diseases [3], it is still unknown how the autonomic nervous system influences the self similarity of blood pressure and heart rate.

To investigate this issue, our work evaluates the possible effects of selective sympathetic and parasympathetic autonomic blockades on self-similarity scaling exponents of blood pressure and heart rate in resting young healthy volunteers.

\section{Methods}

\subsection{Selection of a self-similarity estimator}

The definition of signal self-similarity states that if $x(t)$ is self-similar, then it has the same statistical properties of $a^{-H} x(a t)$, for any $a$ greater than zero. Thus evaluating the self-similarity of $x(t)$ implies the estimation of its scaling exponent $H$. If $x(t)$ is fractional Brownian noise (fBn), then $H$ (also called the Hurst exponent) ranges between 0 and 1 , with $H=0.5$ for white noise.

Several methods have been proposed for estimating the Hurst exponent. A popular algorithm is based on the computation of the slope $\beta$ of the " $1 / \mathrm{f}$ " trend of the power spectrum [4]. The scaling exponent is derived from $\beta$ as $H=(\beta+1) / 2$. This method, however, has some practical limitations when applied to real blood pressure and heart rate data. In fact, the frequency band where the spectra show a " $1 / \mathrm{f}$ " trend is limited to the lower frequencies and should be selected accurately. Moreover, long segments of data are needed to reliably estimate the lower spectral components: but since recordings of long duration may be affected by nonstationarities, the spectral shape may be distorted. For these reasons, we preferred to consider different $H$ estimators.

A more robust estimator is given by the "aggregated variance" algorithm [5]. If $x(t)$ is a $\mathrm{fBn}$ with variance $\sigma_{0}^{2}$, then the variance of the average of $\mathrm{k}$ consecutive signal samples is $\sigma_{\mathrm{k}}{ }^{2}=\mathrm{k}^{2 H-2} \sigma_{0}^{2}$. Therefore $H$ can be derived from the slope of the regression line between $\sigma_{\mathrm{k}}{ }^{2}$ and $\mathrm{k}$ plotted on a log-log scale. This method seems appropriate to also analyze relatively short segments of data. However, it has been proposed for $\mathrm{fBn}$ processes, and its application to 
self-similar processes with scaling exponent greater than 1 might be inappropriate.

A method proposed for the analysis of physiological data, which can provide estimates of scaling exponent greater than 1, is the Detrended Fluctuation Analysis (DFA) algorithm [6]. Briefly, $x(t)$ is integrated and divided into blocks of size k. Then $F(k)$, i.e., the root mean square of the series detrended in each box, is computed. The slope of the regression line between $\log (F(k))$ and $\log (k)$ is taken as an estimate of $H$.

To select a proper estimator for our data, we compared the aggregated variance and DFA algorithms by means of simulated signals. We generated $\mathrm{fBn}$ with $H$ between 0 and 1 making use of the $w f B m$ routine of Matlab 7 (The MathWorks Inc., Natick, MA). To simulate signals with $H$ between 1 and 1.5 , we integrated the $\mathrm{fBn}$ simulations with $0<H \leq 0.5$, obtaining the corresponding fractional Brownian motions, with scaling exponents equal to $H+1$ (for instance, we obtained the classic Brownian motion with $H=1.5$ by integrating white noise simulations, with $H=0.5$ ). An example of three simulated signals is shown in figure 1.

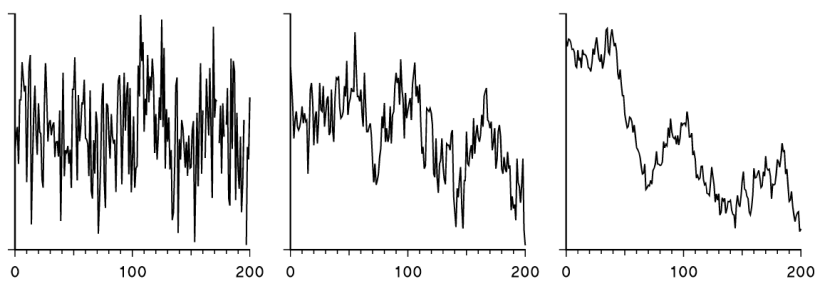

Figure 1. Examples of simulated signals for three different values of the scaling exponent $H$. From left to right: white noise $(H=0.5) ; 1 / \mathrm{f}$ noise $(H=1.0)$; Brownian motion $(H=1.5)$.

Five signals were generated for each $H$ value. Then $H$ was estimated over each group of five simulations by both methods. Results are shown in figure 2 .

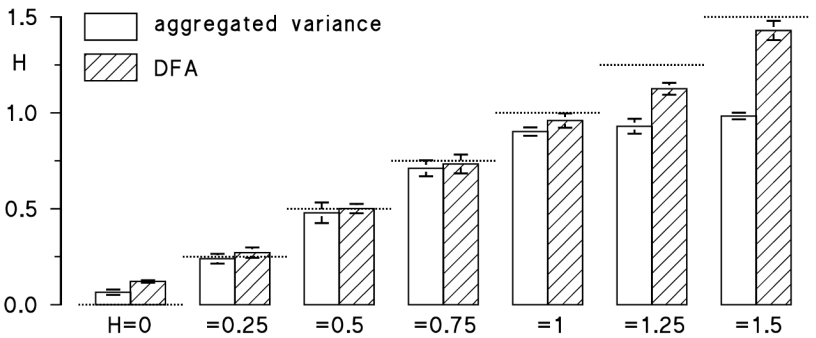

Figure 2. Estimates of $H$ by aggregated variance and DFA for simulated signals with scaling exponent $H$ between 0 and 1.5. The true $H$ values are shown by dotted lines

Both the methods overestimated $H$ when it was equal to 0 . They gave similar estimates when $H$ ranged between 0 and 1 . The bias was lower for the aggregated variance method when $H \leq 0.25$, otherwise it was lower for DFA. When $H>1$, however, only DFA provided reliable estimates. In fact, the aggregated variance method was unable to follow the increase of $H$ from 1 to 1.5 . Since in our study we may expect large changes of $H$ around $H=1$, we selected the DFA algorithm for estimating the scalar exponents of our blood pressure and heart rate data.

\subsection{Data acquisition and analysis}

We recorded ECG and continuous blood pressure noninvasively in 8 healthy subjects during three experimental sessions performed in different days. Each session consisted of 15-20 minutes baseline recording followed by 15-20 minutes recording after administration of a specific drug, as described in [7]. During the recording, the subjects were resting in supine position, in a dim lighted room.
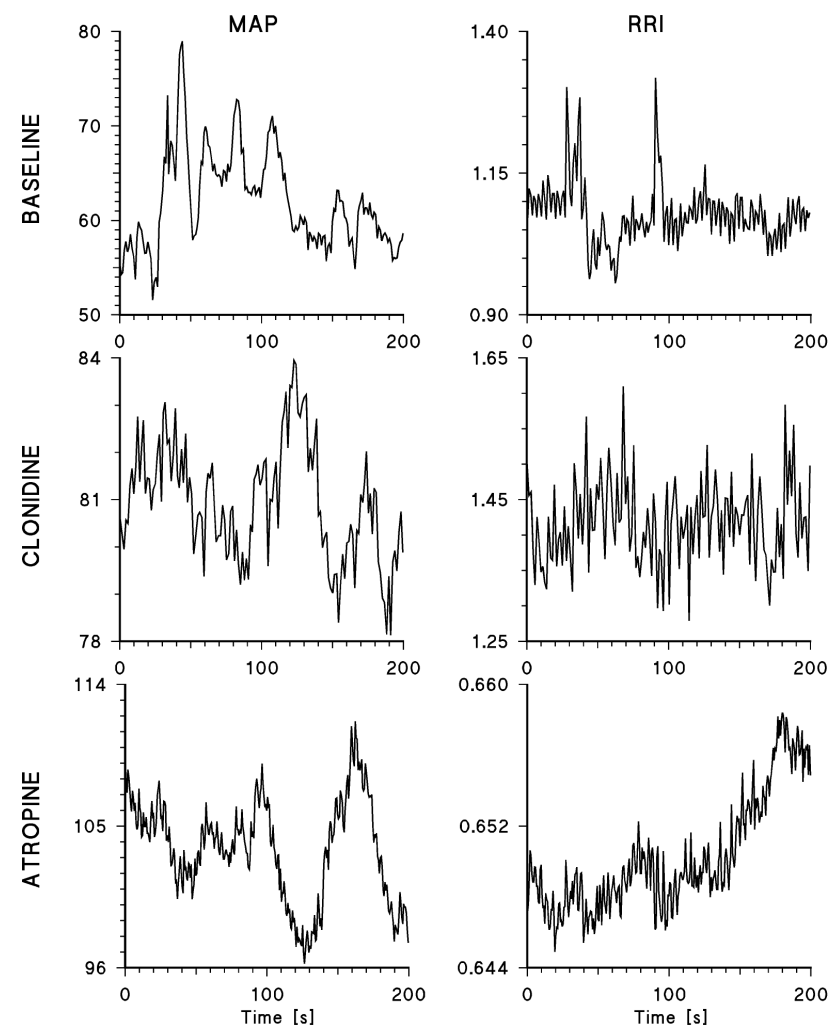

Figure 3. Examples of beat-to-beat time series of MAP and RRI in the same subject during baseline, sympathetic blockade by clonidine and parasympathetic blockade by atropine: values in $\mathrm{mmHg}$ (MAP) and seconds (RRI). Note that the scale of the vertical axis changes in each panel. 
Drugs used to block vagal or sympathetic activities in each of the three sessions were: atropine, a blocker of vagal activity; propranolol, a non specific cardiac $\beta 1$ and vascular $\beta 2$ antagonist; and clonidine, an $\alpha 2$ agonist. Propranolol blocks most of the sympathetic efferences on the heart, and some efferences on the vasculature; while clonidine blocks most of the efferences on both the heart and vasculature. R-R interval, RRI, and mean arterial pressure, MAP, were derived beat-by-beat. Examples of beat-to-beat time series are shown in figure 3.

The self-similarity coefficient of RRI, $\mathrm{H}_{\mathrm{RRI}}$, and MAP, $\mathrm{H}_{\mathrm{MAP}}$, were estimated by DFA. Values were expressed as mean \pm SEM in each condition, and compared by paired ttest. Reproducibility of DFA measures in the three baseline conditions was quantified by the variation coefficient (i.e., the ratio between standard deviation and mean of the three baseline measures, in percent) and analyzed by repeated measures analysis of variance to verify the absence of significant differences among the three measures.

\section{Results}

The three baseline measures were similar ( $p>30 \%$ ) both for $\mathrm{H}_{\mathrm{RRI}}$ and for $\mathrm{H}_{\mathrm{MAP}}$, with variation coefficients equal to $12 \%$ and $8 \%$ respectively. The average \pm SEM over the group of 8 subjects of the three baseline values of $\mathrm{H}_{\text {MAP }}$ was $0.96 \pm 0.02$; this average was significantly greater than the group average of $\mathrm{H}_{\mathrm{RRI}}$ baseline measures, which was equal to $0.75 \pm 0.04(\mathrm{p}<0.002)$.

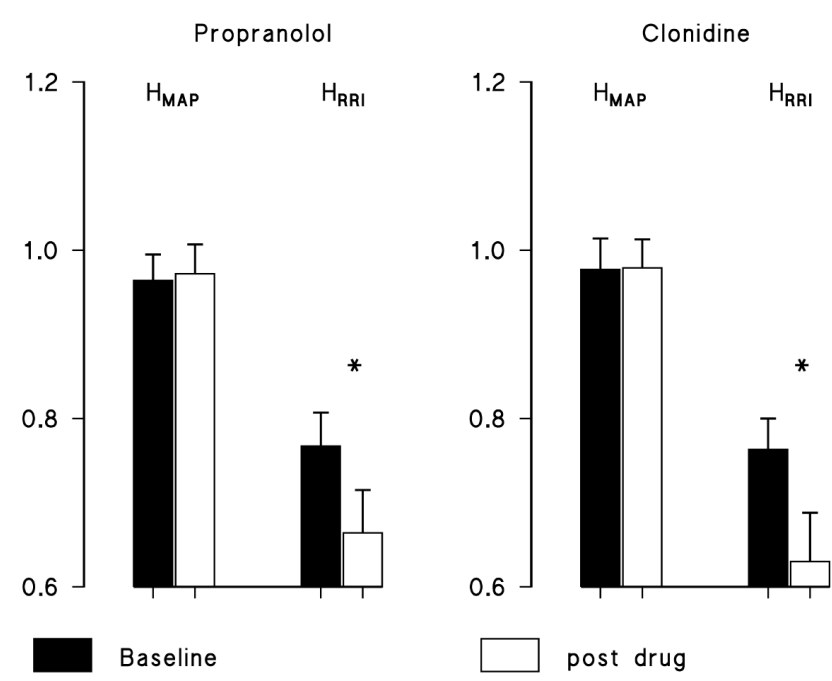

Figure 4. Average scaling exponents of mean arterial pressure, $\mathrm{H}_{\mathrm{MAP}}$, and $\mathrm{R}-\mathrm{R}$ interval, $\mathrm{H}_{\mathrm{RRI}}$, before and after propranolol and clonidine administrations; $* \mathrm{p}<0.05$.

Effects of sympathetic blockades are shown in figure 4. Although the two drugs act differently, their effects were similar. Both the blockades did not alter the selfsimilarity coefficient of blood pressure, while they significantly reduced $\mathrm{H}_{\mathrm{RRI}}$ to value close to those characterizing white noise.

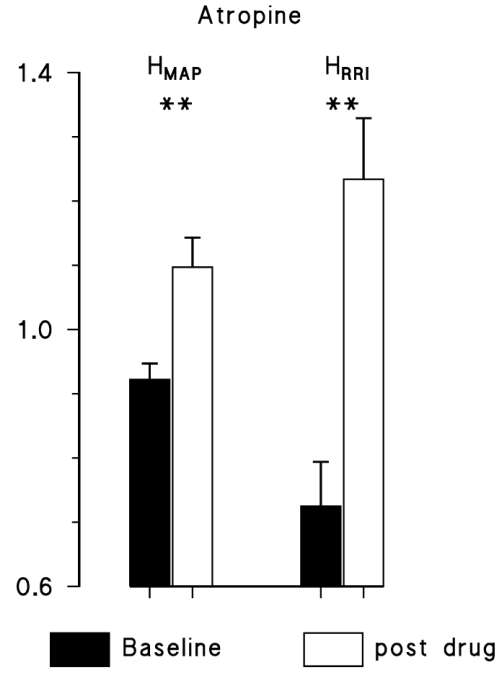

Figure 5. Scaling exponents of MAP and RRI before and during vagal blockade; $* * \mathrm{p}<0.01$.

Figure 5 shows the effects of vagal blockade. Atropine significantly increased both $\mathrm{H}_{\mathrm{MAP}}$ and $\mathrm{H}_{\mathrm{RRI}}$. Their average values were both greater than 1 during atropine administration.

\section{Discussion and conclusions}

This study showed that the scaling structures of blood pressure and heart rate are different, and that the autonomic nervous system differently influences their self-similarity.

Our results suggest that the vagal contribution to the heart rate self-similarity is to add a so-called "antipersistent" component, i.e., a component which makes more probable that a long heart beat is followed by a short beat, and vice versa. This behavior is characterized by $H$ values lower than 0.5 , and thus the vagal contribution would decrease the overall scaling exponent of RR interval. Actually, removal of the vagal contribution by atropine increased $\mathrm{H}_{\mathrm{RRI}}$. It is worth noting that, after atropine infusion, $\mathrm{H}_{\mathrm{RRI}}$ reached values close to those observed in heart failure [3], a disease characterized by a marked reduction in vagal tone [8]. The parasympathetic system seems to provide a similar antipersistent contribution also to the blood pressure dynamics, because also $\mathrm{H}_{\mathrm{MAP}}$ increased after the vagal blockade. The increase was lower than the increment observed for $\mathrm{H}_{\mathrm{RRI}}$, and this may be explained by the lower influence of vagal control on blood pressure.

The sympathetic contribution to the heart rate self- 
similarity seems to have opposite characteristics. Both types of sympathetic inhibition significantly decreased the heart rate scaling exponent, making the heart rate dynamics more similar to a white noise process. This would suggest that the sympathetic contribution we removed with propranolol or clonidine, was responsible for long-term, positive correlations. Thus sympathetic regulation seems to add a "persistent behaviour", or longterm correlation, i.e., a component of variability with $H$ consistently greater than 0.5 .

Surprisingly, the sympathetic system seems to have a negligible influence on blood pressure self-similarity, because this latter did not change after blockade of sympathetic activity. To interpret this result, however, it should be also considered that the baseline condition was characterized by a very low sympathetic tone in our healthy volunteers at supine rest in a dim lighted room.

Baseline data revealed significant differences in the scaling structure of blood pressure and heart rate. The higher scaling exponent observed for MAP indicates that long term correlations weight more in beat-to-beat blood pressure dynamics than in heart rate dynamics. This might depend on the vagal "anti-persistent" contribution, which is greater in heart rate than in blood pressure, and which therefore tends to decrease $\mathrm{H}_{\text {RRI }}$ more than $\mathrm{H}_{\mathrm{MAP}}$.

A final comment regards the methodology for estimating the scaling exponent of cardiovascular data. Not all the estimators proposed in literature provide reliable measures when the scaling exponent is greater than 1. Our study showed that the autonomic nervous system may influence the self-similarity so importantly that the scaling exponent may shift over a wide range of values, from those characterizing white noise $(0.5)$ to those typical of Brownian motion (1.5). For this reason, methods able to reliably estimate self-similarity in the range $0.5 \leq H \leq 1.5$ should be chosen.

\section{References}

[1] Goldberger AL. Fractal mechanisms in the electrophysiology of the heart. IEEE Eng Med Biol Mag 1992; 11(2):47-52.

[2] Goldberger AL. Non-linear dynamics for clinicians: chaos theory, fractals, and complexity at the bedside. Lancet 1996; 347(9011):1312-1314.

[3] Goldberger AL, Amaral LA, Hausdorff JM, Ivanov PC, Peng CK, Stanley HE. Fractal dynamics in physiology: alterations with disease and aging. Proc Natl Acad Sci U S A 2002; 99 Suppl 1:2466-2472.

[4] Schepers HE, van Beek JHGM, Bassingthwaighte JB. Four methods to estimate the fractal dimension from self-affine signals. IEEE Eng Med Biol Mag. 11:57-64, 1992.

[5] Bassingthwaighte JB, Raymond GM. Evaluation of the dispersional analysis method for fractal time series. Ann Biomed Eng 23: 491-505, 1995

[6] Peng CK, Havlin S, Stanley HE, Goldberger AL. Quantification of scaling exponents and crossover phenomena in nonstationary heartbeat time series. Chaos 1995; 5(1):82-87.

[7] Parlow J, Viale JP, Annat G, Hughson R, Quintin L. Spontaneous cardiac baroreflex in humans. Comparison with drug-induced responses. Hypertension 1995; 25(5):1058-1068

[8] Saul JP, Arai Y, Berger RD, Lilly LS, Colucci WS, Cohen RJ. Assessment of autonomic regulation in chronic congestive heart failure by heart rate spectral analysis. Am J Cardiol 1988; 61(15):1292-1299.

Address for correspondence

Paolo Castiglioni

Centro di Bioingegneria, Fondazione Don C. Gnocchi

via Capecelatro 66,

I 20148 Milano

Italy

pcastiglioni@cbi.dongnocchi.it 